\title{
MicroRNAs and Chronic Inflammation Contribution to Gastrointestinal Integrity
}

\author{
A. Ptak-Belowska*, A. Targosz and T. Brzozowski \\ Department of Physiology, Jagiellonian University Medical College, Cracow, Poland
}

\begin{abstract}
Recent studies have revealed that chronic inflammation represents a major basis for different forms of human malignancies. Chronic inflammations are involved in the pathogenesis of $15-25 \%$ of human malignancies. Gastrointestinal (GI) cancer is one of the most common causes of mortality in the European Union. The mechanisms leading to cancer development and its progression are not completely understood. Advances are required both in early detection and therapy of GI cancers. There are many factors connecting inflammation and cancer. Cytokines that are small protein molecules regulating growth, differentiation, development and immune response mechanisms in cells. Overexpression of cyclooxynenase-2 is associated with decreased apoptosis, cell to cell adhesion, increased proliferation and angiogenesis contributes to the increased immunosuppresion and mediates carcinogenetic effects. MicroRNAs are regarded as a novel class of gene expression regulators. They are gene-silencing RNAs which negatively regulate gene expression. After binding to target mRNAs they lead either to mRNA destruction or inhibition of translation. Hence, they can play an important role in carcinogenesis. Currently, almost all of the miRNA-related studies on cancers based on the different expression profile of miRNAs in cancer cells compared to normal cells.
\end{abstract}

In summary, miRNAs, proinflammatory cytokines and other factors, may be involved in cancer development based on chronic inflammation by controlling cell differentiation and apoptosis. Assessing the role of miRNAs will provide the new insights on their contribution to the link between chronic inflammation and subsequent cancer, and new markers for cancer diagnoses and cancer therapy.

Keywords: Cancer, chronic inflammation, cytokines, differentiation, gene, microRNAs.

Recent studies have revealed that chronic inflammation represents a major basis for different forms of human malignancies. It was Rudolf Virchov in 1863, who for the first time proposed the role of inflammation in carcinogenesis as he noticed the presence of leukocytes in cancer tissues [1]. Chronic inflammations are involved in the pathogenesis of $15-25 \%$ of human malignancies [1, 2]. Chronic inflammation that increased cancer risk comprise microbial infections like Helicobacter pylori (H. pylori) infection for gastric cancer, and autoimmune diseases (inflammatory bowel disease for colon cancer or Hashimoto thyroiditis for thyroid papillary carcinoma) [2,3]. Gastrointestinal (GI) cancer is one of the most common causes of mortality in the European Union (EU) with $25 \%$ of mortality attributable to cancer and half of this due to GI cancer. The mechanisms leading to cancer development and its progression are not completely understood. Advances are required both in early detection and therapy of GI cancers. On the other hand, thyroid cancer is the most frequent endocrine neoplasia with rapidly increasing occurrence [3].

The possible mechanisms explaining how inflammation can contribute to carcinogenesis contain:

- Induction of genomic instability,

- Epigenetic changes resulting in inappropriate gene expression,

- Resistance to apoptotic signals,

- Increased proliferation and tumour vascularisation,

- Overexpression of proinflammatory cytokines, cyclooxygenase-2 (COX-2), nitric oxide (NO) promoting tumour progression in its microenvironment,

- Stromal cells in surrounding tissue and infiltrated immune cells activating proinflammatory cytokines,

- MicroRNAs (miRNAs).

In physiological conditions an inflammatory process act as a host defence against injury and is a self-limiting process [4]. However, inadequate and chronic inflammation often leads to cancers.

*Address correspondence to this author at the Department of Physiology, Jagiellonian University Medical College, 31-531Cracow, 16 Grzegorzecka Street, Poland; Tel: +48124211006; Fax: +4812 422-20-14; E-mail: agata.ptak-belowska@uj.edu.pl
Table 1. Examples of Chronic Inflammation Leading to Cancer

\begin{tabular}{|c|c|}
\hline Inflammation & Cancer \\
\hline \hline Chronic pancreatitis & Pancreatic cancer \\
\hline Barrett's esophagitis & Esophageal cancer \\
\hline Inflammatory bowel disease & Colorectal cancer \\
\hline Chronic gastritis (Helicobacter pylori) & Gastric cancer \\
\hline Thyroiditis & Thyroid carcinoma \\
\hline Infection with HBV, HCV & Hepatocellular carcinoma \\
\hline
\end{tabular}

In response to proinflammatory stimuli immune cells can generate reactive oxygen species (ROS) and reactive nitrogen species (RNS) [23]. Both ROS and RNS can act as effectors in inflammatory-related carcinogenesis [1-4]. Overproduction of ROS and RNS in the inflamed tissue leads to subsequent DNA damage: activation of oncogenes or inactivation of tumour suppressor genes [5]. Moreover ROS-induced DNA damages contain DNA strand breaks and DNA modifications resulting in replication errors contributing to cancer onset [6]. NO exerts its effect in inflammation-related carcinogenesis by direct modification of DNA. NO was also found to inactivate DNA repair enzymes [7].

The genomic DNA can be affected not only by direct mutational changes but also by epigenetic variations including DNA methylation and histone modifications [8]. DNA-methylation is the covalent addition of a methyl group to the 5-position of cytosine in the DNA [1]. DNA methylation is a critical epigenetic control of gene expression. The principal abnormal DNA methylation is hypermethylation that usually occurs in the promoter regions of tumour suppressor genes [8, 9]. Promoter hypermetylation of tumour suppressor genes i.e.: p16, p73, p53 results in transcriptional silencing $[8,10]$. Therefore hypermethylation of promoter sides leads to epigenetic silencing of tumour suppressor genes. As a result disturbances in cell cycle control, DNA repair, and cell adhesion can be observed and these processes are regarded to be crucial in carcinogenesis [9]. There are studies showing, that hypermethylation of E-cadherin gene in intestinal metaplasia in patients infected with $H$. pylori can be regarded as an early event in gastric cancer developing [11]. What's more there are studies confirming that $H$. pylori infection is responsible for DNA hypermethylation of tumour suppressor p16 gene [12]. This action strongly suggests impact of epigenetic changes in inflammatory-related cancers. 
Other epigenetic mechanism regarded to control gene expression is chromatin remodelling by means of histone modification. Two enzymes: histone deacetylase (HDAC) and histone acetyl transferases (HATs) are involved in transcriptional regulation of many cancer-related genes [13]. It was shown that the inflammation-induced changes in histones may by due to an upregulation of proinflammatory cyclooksygenase-2 (COX-2) gene in human bronchial epithelial cells [14]. Moreover, the activation of NF- $\kappa \mathrm{B}$, increased release of IL-6 and IL-8 in human alveolar cells were also related to increased acetylation of histone 4 [15]. Hence, the inflammation-related changes in histones and subsequent upregulation of $\mathrm{NF}-\kappa \mathrm{B}, \mathrm{COX}-2$ and proinflammatory cytokines indicate that inflammation through epigenetic mechanisms may contribute to genetic instability of tumour cells.

\section{FACTORS CONNECTING INFLAMMATION AND CANCER}

\section{Cytokines}

Cytokines are small protein molecules regulating growth, differentiation, development and immune response mechanisms in cells [16]. Cytokine signalling is pleiotropic as cross-regulation of one cytokine by other cytokine exists; different response by the same cytokine depends on a cell type [1]. Moreover, synergistic or antagonistic effects of cytokines are exerted by combine stimulation with other factors. Cytokines play an important role in the communication between cancer cells and surrounding environment (stromal tissue) promoting neoplasia progression [17, 18]

\section{COX-2 and Prostaglandins}

Important enzymes involved in inflammation and tumour growth are cyclooxygenase (COX)-1 and COX-2. There are two isoforms of COX enzymes: constitutive $\mathrm{COX}-1$ which is homeostatically expressed in most tissues and the inducible COX-2 stimulated by various cytokines and growth factors and detectable in the inflamed tissues [19]. Overexpression of COX-2 is associated with decreased apoptosis, cell to cell adhesion, increased proliferation and angiogenesis contributes to the increased immunosuppresion and mediates carcinogenetic effects [19,20].

\section{iNOS and Nitric Oxide (NO)}

NO which is produced during arginine metabolism by different isoforms of NOS is an important factor connecting inflammation and cancer. During inflammation inducible form of NOS (iNOS)

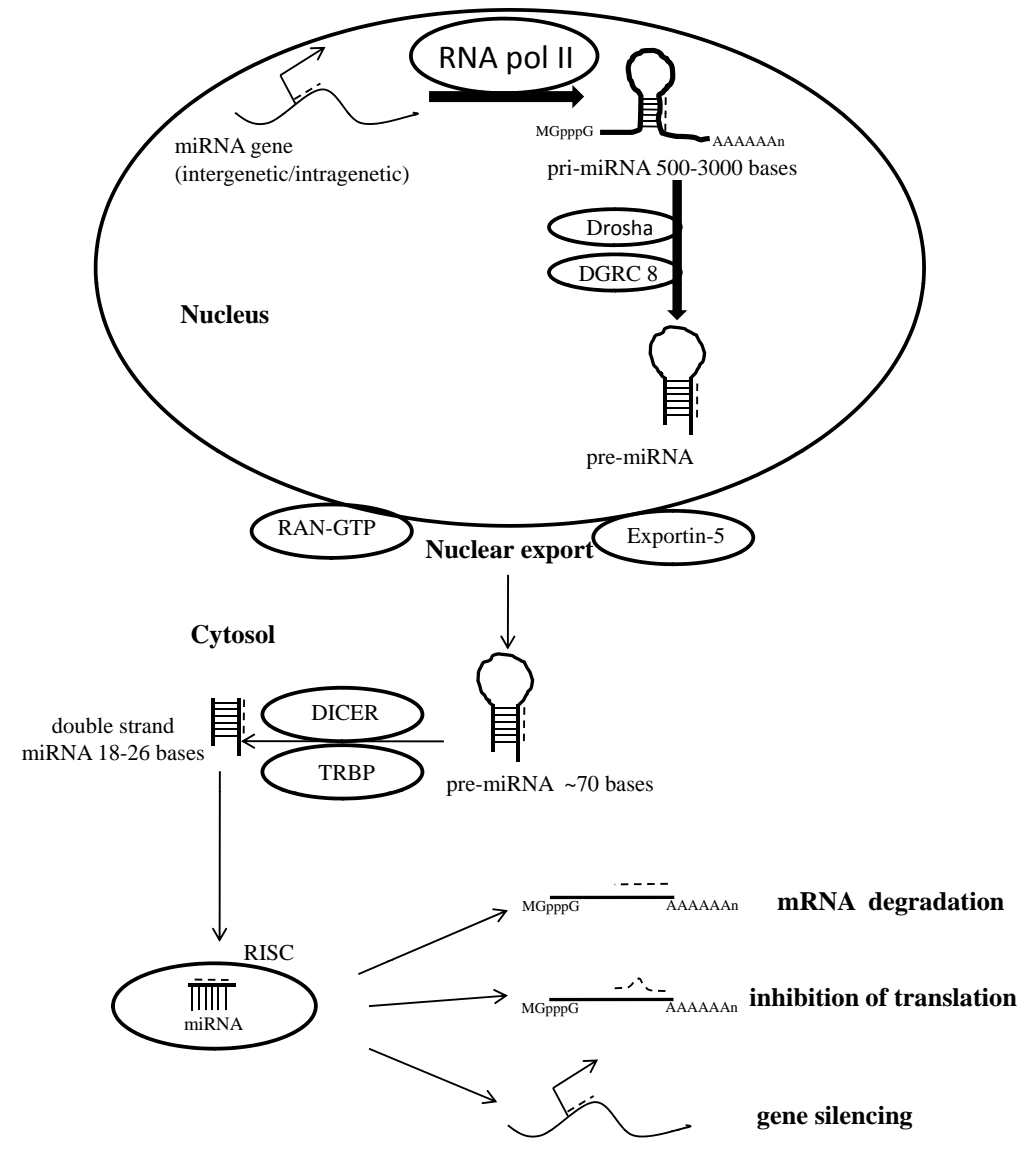

\section{MicroRNA biogenesis}

Fig. (1). MicroRNA biogenesis. Pri-miRNA transcripts are processed into pre-miRNAs by an enzymatic complex that includes the nuclear RNAse III enzyme Drosha and DGRC8. The resulting pre-miRNA is transported to the cytoplasm by exportin-5 and a Ran GTPase. Once in the cytoplasm, pre-miRNAs are processed into 22-nucleotide duplexes by another RNAse III enzyme, Dicer, in association with TRBP. The strand corresponding to the mature miRNA is subsequently loaded onto the RISC. Mature miRNAs bind the 3'-untranslated region of target mRNAs and subsequently destabilize them, block their translation, or both.

Abbreviations: DGRC8, DiGeorge syndrome critical region 8; mRNA, messenger RNA; miRNA, microRNA; pre-miRNA, precursor miRNA; pri-miRNA, primary miRNA; Ran, RAS-like nuclear protein; RISC, RNA-induced silencing complex; TRBP, trans-activation-response RNA-binding protein. 
leads to production of NO [1, 21]. It is known that iNOS expression is regulated by several proinflammatory cytokines and it functions to generate high levels of NO via oxidative metabolism of $\mathrm{L}$ arginine. NO seems to have a dual role in colitis. On one hand, NO leads to vasodilatation, host defence and inhibition of neutrophil activation and platelet aggregation. On the other hand, NO is a highly reactive free radical and may rapidly react with active oxygen to generate peroxynitrate, which causes severe detrimental effects on colonic mucosa [21]. Moreover, it has been shown, that the level of NO and the expression on iNOS are elevated in numerous pre-cancer and neoplastic lesions [22].

\section{NF-кB}

A large number of DNA-binding proteins are activated in response to inflammation [1,3]. They can cause aberrant induction of numerous genes in neoplastic cells and in surrounding tissue cells. NF- $\kappa B$, a transcriptional factor, plays a crucial role in carcinogenesis, because its inproper activation contributes to tumorigenesis $[1,6]$. Incorrect activation of $\mathrm{NF}-\kappa \mathrm{B}$ leads to transactivation of target genes: inflammatory (e.g. COX-2, iNOS), anti-apoptotic (e.g. XIAP, Bcl-2), cell cycle regulating (cyclin D1) [1]. Therefore, NF- $\kappa \mathrm{B}$ has been recently identified as a crucial factor linking inflammation and cancer [24].

\section{MiRNAs in Cancer Promotion}

MiRNAs are regarded as a novel class of gene expression regulators. The miRNAs are a family of non-coding, small size (1922 oligonucleotides) RNA fragments. They are gene-silencing RNAs which negatively regulate gene expression [25, 26]. After binding to target mRNAs they lead either to mRNA destruction or inhibition of translation [27]. There are studies suggesting that miRNAs can play an important role in carcinogenesis [25] because quite a lot of miRNAs have already been shown to act as oncogenes or tumour suppressor genes in numerous malignancies [28]. Currently, almost all of the miRNA-related studies on cancers based on the different expression profile of miRNAs in cancer cells compared to normal cells.

The alterations in miRNA expression may play an important role in many diseases including: colorectal neoplasia (miR-143, miR-145), hepatocellular carcinoma (miR-18, miR-224, miR-199, miR-195, miR-200, miR-125), lung cancer (miR-17-92), papillary thyroid carcinoma (miR-221, miR-222, miR-146, miR-181), testicular germ cell tumours (miR-372, miR-373) [28, 33]. Due to its role in carcinogenesis miRNAs are also referred to as "oncomiRNas" [29, 30, 31,33] MicroRNAs are regarded to influence on cell proliferation, apoptosis, and metabolism and play a crucial role in chronic inflammation often leading to cancer [33]. Recently it was shown, that chronic inflammatory bowel diseases such as ulcerative colitis (UC) are associated with differential expression of genes involved in inflammation and tissue remodelling including miRNAs [34]. Decreased expression of miR192, was observed in active UC promoting colon cancer [34]. Therefore, expression profiles of specific miRNAs may be diagnostic and prognostic markers of many diseases including chronic inflammatory diseases leading to cancers. Because the role of miRNA in contribution to the link between inflammation and cancer has just been started to investigate uncovering the role of specific "oncomiRNas" seems to have promise in future research.

Lately, miRNAs have been also studied as potential diagnostic or therapeutic targets in cancer treatment [35]. An association has been reported between miRNA expression in tumours and chemoand radio sensitivity. Dissimilar miRNAs have been found to predict sensitivity to anticancer treatment e.g.: miR-30c, miR-130a and miR-335 are downregulated in various chemoresistant cell lines [35]. Therefore the role of miRNAs in carcinogenesis and on modification of anticancer treatment needs further investigation in order to asses the possible intracellular pathways involved in these processes.

In summary, miRNAs, proinflammatory cytokines and other factors, may be involved in cancer development based on chronic inflammation by controlling cell differentiation and apoptosis. However, some miRNAs may be concerned in carcinogenesis by targeting cancer oncogenes and tumour suppressors. Assessing the role of miRNAs will provide the new insights on their contribution to the link between chronic inflammation and subsequent cancer, and new markers for cancer diagnoses and cancer therapy.

\section{REFERENCES}

[1] Balkwill, F.; Msantovani, A.Inflammation and cancer: back to Virchov? Lancet, 2001, 539-545.

[2] Allavena, P.; Garlanda, C.; Borello, M.G.; Sica, A.; Mantovani, A. Pathways connecting inflammation and cancer. Curr. Opin. in Genetics and Development, 2008, 18, 3-10.

[3] Guarino, V.; Castellone, M.D.; Avilla, E.; Melillo, R.M. Thyroid cancer and inflammation. Moll. Cell Endocrinology, 2010, 321, 94-102.

[4] Jackson, L.; Evers, B.M. Chronic inflammation and pathogenesis of GI and pancreatic cancers. Cancer Treat. Res., 2006, 130, 39-65.

[5] Schottenfeld, D.; Beebe-Dimmer, J. Chronic inflammation: a common and important factor in the pathogenesis of neoplasia. CA Cancer J.Clin., 2006, 56, 69-83.

[6] Cooke, M.S.; Evans, M.D.; Dizdaroglu, M.; Lunec, J. Oxidative DNA damage: mechanisms, mutation and disease. FASEB J., 2003, 17, 1195-1214. Jaiswal, M.; LaRusso, N.F.; Burgart, L.J.; Gores, G.J. Inflammatory cytokines induce DNA damage and inhibit DNA repair in cholangiocarcinoma cells by a nitric-oxide- dependent mechanism. Cancer Res., 2000, 60, 184-190.

[8] Herceg, Z. Epigenetics and cancer: towards an evaluation of the impact of environmental and dietary factors. Mutagenesis, 2007, 22, 91-103.

[9] Tischoff, I.; Wittekind, C.; Tannapfel, A. Role of epigenetic alterations in cholangiocarcinoma. J Hepatobiliary Pancreat. Surg., 2006, 13, 274-279.

[10] Ushiku, T.; Chong, J.M.; Uozaki, H.; Hino, R.; Chang, M.S.; Sudo, M.; Rani, B.R.; Sakuma, K,; Nagai, H.; Fukuyama, M. p73 gene promoter methylation in Epstein-Barr virus-associated gastric carcinoma. Int. J. Cancer, 2007, 120, 60-66.

[11] Chan, A.O.; Lam, S.K.; Wong, B.C.; Wong, W.M.; Yuen, M.F.; Yeung, Y.H.; Hiu, W.M.; Rashid, A.; Kwong, Y.L. Promoter methylation of Ecadherin gene in gastric mucosa associated with Helicobacter pylori infection and in gastric cancer. Gut, 2003, 52, 502-506.

[12] Maekita, T.; Nakazawa, K.; Mihara, M.; Nakajima, T.; Yanaoka, K.; Iguchi, M.; Arii, K. High levels of aberrant DNA methylation in Helicobacter pyloriinfected gastric mucosae and its possible association with gastric cancer risk. Clin .Cancer Res., 2006, 12, 989-995.

[13] Huang, L. Targeting histone deacetylases for the treatment of cancer and inflammatory diseases. J. Cell Physiol., 2006, 209, 611-616.

[14] Cao, D.; Bromberg, P.A.; Samet, J.M. COX-2 expression induced by diesel particles involves chromatin modification and degradation of HDAC1. Am. J. Respir. Cell Mol. Biol., 2007, 37, 232-239.

[15] Moodie, F.M.; Marwick, J.A.; Anderson, C.S.; Szulakowski, P. Oxidative stress and cigarette smoke alter chromatin remodelling but differentially regulate NF- $\mathrm{KB}$ activation and proinflammatory cytokine release in alveolar epithelial cells. FASEB J., 2004, 18, 1897-1899.

[16] Lu, H.; Ouyang, W.; Huang, C. Inflammation, a key event in cancer development. Mol. Cancer Res., 2006, 4, 221-233.

[17] Ben-Baruch, A. Host microenvironment in breast cancer development: inflammatory cells, cytokines and chemokines in breast cancer progression: reciprocal tumor-microenvironment interactions. Breast Cancer Res., 2003, 5, 31-36.

[18] Porta, C.; Subhra Kumar B.; Larghi, P.; Rubino, L.; Mancino, A.; Sica, A Tumor promotion by tumor-associated macrophages. Adv. Exp. Med. Biol., 2007, 604, 67-86.

[19] Brzozowski, T.; Konturek, P.C.; Pajdo, R.; Ptak-Belowska, A.; Kwiecien, S.; Pawlik, M.; Drozdowicz, D.; Sliwowski, Z.; Brzozowski, B.; Konturek, S.J.; Pawlik, W.W. Physiological mediators in nonsteroidal anti-inflammatory drugs (NSAIDs)-induced impairment of gastric mucosal defense and adaptation. Focus on nitric oxide and lipoxins. J. Physiol. Pharmacol., 2008, 2, 89-102.

[20] Majka, J.; Rembiasz, K.; Migaczewski, M.; Budzynski, A.; Ptak-Belowska, A.; Pabianczyk, R.; Urbanczyk, K.; Zub-Pokrowiecka, A.; Matlok, M.; Brzozowski, T. Cyclooxygenase-2 (COX-2) is the key event in pathophysiology of Barrett's esophagus. Lesson from experimental animal model and human subjects. Physiol. Pharmacol., 2010, 4, 409-18.

[21] Konturek, P.C.; Brzozowski, T.; Engel, M.; Burnat, G.; Gaca, P.; Kwiecien, S.; Pajdo, R.; Konturek, S.J. Ghrelin ameliorates colonic inflammation. Role of nitric oxide and sensory nerves. J. Physiol. Pharmacol., 2009, 60, 41-7. 
[22] Jaiswall, M.; LaRusso, N.F.; Gores, G.J. Nitric oxide in gastrointestinal epithelial cell carcinogenesis: linking inflammation to oncogenesis. Am. J. Physiol. Gastrointest. Liver Physiol., 2001, G626-G634.

[23] Bandyopadhyay, D.; Ghosh, G.; Bandyopadhyay, A.; Reiter, R.J. Melatonin protects against piroxicam-induced gastric ulceration. J. Pineal. Res., 2004, 3, 195-203.

[24] Pikarsky, E.; Porat, R.M.; Stein, I.; Abramovitch, R.; Amit, S.; Kasem, E. $\mathrm{NF}-\kappa \mathrm{B}$ function as a tumour promoter in inflammation-associated cancer. Nature, 2004, 431, 590-597.

[25] Lee, Y.; Kim, M.; Han, J.; Yeom, K.H.; Lee, S.; Baek, S.H.; Kim, V.N. MicroRNA genes are transcribed by polymerase II. EMBO J., 2004, 40514060 .

[26] Osada, H.; Takahashi, T. MicroRNAs in biological processes of carcinogenesis. Carcinogenesis, 2007, 28, 2-12.

[27] Valencia-Sanchez, M.A.; Liu, J.; Hannon, G.J.; Parker, R. Control of translation and mRNA degradation by miRNAs and siRNAs. Genes Dev., 2006, 20, 515-524.

[28] Giannakakis, A.; Coukos, G.; Hatzigeorgiou, A.; Sandaltzopolus, R.; Zhang, L. miRNA genetic alterations in human cancers. Expert. Opin. Biol. Ther, 2007, 1375-1386.

[29] Cho, W.C. OncomiRs: the discovery and progress of microRNAs in cancers. Mol. Cancer, 2007, 6, 60 .
Esquela-Kerscher, A.; Slack, F.J. Oncomirs-microRNAs with a role in cancer. Nat. Rev .Cancer, 2006, 6, 259-269.

O’Connel, R.M.; Taganov, K.D.; Boldin, M.P.; Cheng, G.; Baltimore, D. MicroRNA-155 is induced during the macrophage inflammatory response. Proc. Natl. Acad. Sci. USA, 2007, 104, 1604-1609.

Oshima, H.; Sawa, T.; Akaike, T. 8-Nitroguanine, a product of nitrative DNA damage caused by reactive nitrogen species: formation, occurrence, and implications in inflammation and carcinogenesis. Antioxid. Redox. Signal., 2006, 8, 1033-1045

Slattery, M.L.; Wolff, E.; Hoffman, M.D.; Pellatt, D.F.; Milash, B.; Wolff, R.K. MicroRNAs and colon and rectal cancer: Differential expression by tumor location and subtype. Genes Chromosomes Cancer, 2011, 3, 196-206. Wu, F.; Zikusoka, M.; Trindade, A.; Dassopoulos, T.; Harris, M.L.; Bayless, T.M.; Brant, S.R.; Chakravarti, S.; Kwon, J.H. MicroRNAs are differentially expressed in ulcerative colitis and alter expression of macrophage inflammatory peptide-2 alpha. Gastroenterology. 2008, 5, 1624-1635.

Hummel, R.; Hussey, D.J.; Haier, J. MicroRNAs: predictors and modifiers of chemo- and radiotherapy in different tumour types. Eur. J. Cancer, 2010, 46, 298-311. 